Marquette University

e-Publications@Marquette

Nursing Faculty Research and Publications

Nursing, College of

2-1-2011

\title{
Characteristics of the Menstrual Cycle After Discontinuation of Oral Contraceptives
}

Claudia L. Nassaralla

Marquette University

Joseph B. Stanford

University of Utah

Diane Daly

Saint John's Mercy Medical Center

Mary Schneider

Marquette University, mary.schneider@marquette.edu

Karen C. Schliep

University of Utah

See next page for additional authors

Accepted version. Journal of Women's Health, Vol. 20, No. 2 (February 2011). DOI. (c) 2011 Mary Ann Liebert, Inc. Used with permission. 
Authors

Claudia L. Nassaralla, Joseph B. Stanford, Diane Daly, Mary Schneider, Karen C. Schliep, and Richard Fehring 


\title{
Characteristics of the Menstrual Cycle After Discontinuation of Oral Contraceptives
}

Claudia L. Nassaralla, Ph.D., M.D., Joseph B. Stanford, M.D., MSPH, K. Diane Daly, R.N., CFCE, Mary Schneider, M.S.N., R.N., Karen C. Schliep, MSPH, and Richard J. Fehring, Ph.D., R.N.

\begin{abstract}
Background: Menstrual cycle function may continue to be altered after discontinuation of oral contraceptives (OC). Few studies have been published on the effects of recent OC use on menstrual cycle parameters; none have examined characteristics of the menstrual flow or the quality of cervical mucus. The purpose of this retrospective matched cohort study is to assess biomarkers of the menstrual cycle after discontinuation of OCs.
\end{abstract}

Methods: Among a sample of women who daily recorded observations of menstrual cycle biomarkers, 70 women who had recently discontinued OCs were randomly matched by age and parity with 70 women who had not used OCs for at least 1 year. Outcomes investigated included overall cycle length, length of the luteal phase, estimated day of ovulation, duration of menstrual flow, menstrual intensity, and mucus score. Differences between recent $\mathrm{OC}$ users and controls were assessed using random effects modeling.

Results: Recent OC users had statistically significantly lower scores for mucus quality for cycles 1 and 2. Additionally, OC users had a later estimated day of ovulation that was statistically significant in cycle 2 and a decreased intensity of menstrual flow that was significant in the first four cycles (difference $=-0.48$ days). In random effects modeling, all these parameters were significantly different for the first six cycles combined.

Conclusions: Menstrual cycle biomarkers are altered for at least two cycles after discontinuation of OCs, and this may help explain the temporary decrease in fecundity associated with recent OC use. 


\section{Introduction}

Oral contraceptives $(\mathrm{OC})$ are the most commonly used reversible method of contraception among women between the ages of 15 and 44 in the United States. ${ }^{1}$ Many of these women will eventually discontinue use of OCs in order to achieve a pregnancy. In addition, some will use fertility awareness methods to enhance their chances to conceive. ${ }^{2}$ Accurate knowledge of the parameters of the menstrual cycle is important for couples trying to achieve pregnancy as well as for couples using natural methods to avoid pregnancy. Therefore, it is of interest to know if the menstrual cycle may continue to be altered for some time after discontinuation of OCs.

Few studies have been published on the effects of recent use of OCs onmenstrual cycle parameters. ${ }^{3-5}$ One study compared 175 women discontinuing OCs with 284 similar women who never used OCs, where both groups of women charted basal body temperatures (BBT) and cervical mucus. ${ }^{5}$ In that study, the recent OC users had significantly longer cycles than the control women. The researchers concluded that although cycle disturbances after discontinuation of OCs were reversible, the time required for cycles to normalize was $\geq 9$ months. Furthermore, only $57.9 \%$ of the women in the study had an ovulatory cycle with the luteal phase judged sufficient to achieve pregnancy in their first cycle after OC discontinuation (based on thermal shift). They theorized that the delayed normalization of the parameters of the menstrual cycle was due to the time necessary for the hypothalamic-pituitary-ovarian axis to normalize after being influenced by the exogenous hormones from the OCs. This study did not examine characteristics of the menstrual flow or quality of cervical mucus. ${ }^{5}$

Fertility awareness methods can be used to enhance couples chances to achieve pregnancy. ${ }^{6}$ These methods use one or more of several natural biomarkers [i.e., variations in cervical mucus characteristics, BBT, cervical checks, and home urinary hormonal kits that measure estrone-3-glucuronide (E3G) and luteinizing hormone (LH) levels] to determine the fertile window in the cycle or the days when a couple is likely to conceive. ${ }^{7,8}$ Among these biomarkers, the presence of estrogen-stimulated cervical mucus is particularly relevant to achieve pregnancy. Previous studies determined that estrogenic mucus enhances the sperm motility, storage and transport of normal spermatozoa. ${ }^{9-12}$ Furthermore, cervical mucus discharge methods identify a longer window of fertility than LH kits. ${ }^{13}$ Identifying the full window of fertility may lead to higher rates of conception. ${ }^{14}$ Therefore, couples trying to conceive after discontinuation of OCs may benefit by being able to identify the biomarkers of the women's menstrual cycles, particularly the changes in cervical mucus discharge and the time within their 
cycle they are most likely to conceive. This knowledge could enhance their chances to achieve pregnancy.

The purpose of this study was to determine the characteristics of biomarkers of the menstrual cycle [that is, overall menstrual cycle length in addition to estimated length of the luteal phase, duration of menses, estimated day of ovulation (follicular phase length), menstrual flow score, and mucus score] during the first six menstrual cycles after discontinuation of OCs and to compare these with the biomarker characteristics in menstrual cycles of women who had not used OCs for at least 1 year previously.

\section{Materials and Methods}

\section{Design}

This retrospective cohort study compared 70 women who had discontinued OCs within $\leq 12$ weeks (cases) with 70 matched women who had not used OCs for at least 1 year (controls). Both groups of women recorded daily observations of vaginal discharge on charts according to a standardized protocol, and from these charts, we abstracted information for up to six consecutive menstrual cycles for each woman.

\section{Sites}

The 140 women came from three clinical sites in the United States (Atlanta, Georgia; Milwaukee, Wisconsin; St Louis, Missouri). The Atlanta site is a private clinic of an obstetrician and gynecologist; the Milwaukee site is the Natural Family Planning Institute at Marquette University; and the St Louis site is the Department of FertilityCare Services of St. John's Mercy Medical Center. These 140 women were all taught the Creighton Model FertilityCare System (CrMS) by either a physician or a nurse who was certified in the CrMS.

\section{Creighton Model Fertility Care System}

The CrMS is a standardized method of natural family planning (NFP) that includes an introductory session, structured follow-up visits, and daily observation and charting of biomarkers by the woman or couple using the method. Teachers of the CrMS undergo an intensive training program that lasts 18 months and an external certification process.

The CrMS teaches women how to monitor and record biomarkers of the menstrual cycle by daily observations of vaginal discharge according to standardized protocols (an example of a CrMS menstrual cycle chart is shown in Fig. 1). From the CrMS daily diary or chart, one can monitor and interpret characteristics of vaginal bleeding, the vaginal discharge of cervical mucus, 
the estimated day of ovulation, cycle length, and luteal phase length. ${ }^{15}$ The peak day of estrogenic cervical mucus discharge (i.e., the last day of any mucus that is clear, stretchy, or lubricative) is a fairly accurate indicator of the day of ovulation with an accuracy of 2-3 days. ${ }^{16,17}$ In validation studies with the CrMS specifically, ovulation as assessed by hormonal measures occurred between 2 days before and 1 day after peak day in $89.2 \%$ of cycles and between 3 days before and 3 days after peak day in $100 \%$ of cycles. ${ }^{18}$

All the CrMS charts from the 140 women included in this study are currently housed in secure storage in the Marquette University Institute for Natural Family Planning or at Saint John's Mercy Medical Center. The charts represent menstrual cycles from 1985 through 1995. The retrospective analysis of these data was approved by the Marquette University Office of research compliance, the University of Utah Institutional Review Board for Human Subjects, and the St. John's Mercy Medical Center Institutional Review Board. The database includes no identifying information.

\section{Sample}

Women who were new users of the CrMS and between the ages of 18 and 42 were sampled. Seventy women who had discontinued use of OCs within $\leq 12$ weeks (cases) were randomly matched by age (within 1 year) and parity (parous or nonparous) with 70 women who had not used OCs within the past year (controls). The majority of the women were learning the CrMS to temporarily avoid pregnancy, but pregnancy intention for use of the CrMS was not a selection criterion of this study. Women with a history of infertility and women who were breastfeeding were excluded. There was no exclusion based on cycle length. All inclusion, exclusion, and matching criteria were determined as of the time the woman started charting with the CrMS. Cycles within pairs were matched by sequential cycle number. Because some women had one or more missing cycles, the number of matched cycles available for analysis for any given cycle number was less than the total number ofmatched pairs ofwomen. In the total database, there were 675 cycles; cycles included in the matched pair analysis $(n=230$ matched pairs, $n=460$ cycles) are outlined in Table 1 .

\section{Database}

A database was extracted from the CrMS charts of the sample. Each participant contributed at least one and no more than six cycles to the database, based on the cycles available in her CrMS charts. Demographic information was collected from the standardized general intake form completed by the participant before the first follow-up visit. Additionally, 
satisfaction with and confidence in using the CrMS method was assessed at both the first and sixth follow-up visits by the CrMS instructor. The first and sixth follow-up visits usually occur 2 weeks and 6 months after the client starts charting with the CrMS, respectively. Satisfaction was assessed on a Likert scale (1-5), with $1=$ unsatisfied and $5=$ very satisfied. Confidence was also assessed on a Likert scale (1-5), with $1=$ very unconfident and $5=$ very confident, with an additional category for overconfident. There were very few responses of overconfident, so thesewere collapsed to a response of 5 , very confident.

Potential confounding variables were obtained from the records kept by the CrMS practitioner (teacher) at the first follow-up visit (via the standardized follow-up form) and included medication use (including antibiotics, antihistamines, vitamins, expectorants, and hormones). Medication use was dichotomously coded (consumers, nonconsumers). Further, the length of time the woman had taken OCs and time elapsed since discontinuing OCs were also obtained from the followup form. The biomarkers of menstrual cycle function, abstracted from the participant's standardized CrMS charts, included cycle length, duration of menses, the estimated day of ovulation (EDO) based on the peak day of cervical mucus, the postovulatory (luteal) phase (based on the EDO and cycle length), the menstrual flow score, and the cervical mucus score. To ensure reliability of the dataset, two CrMS trained health professionals read and interpreted each menstrual cycle chart and independently validated the data entries.

\section{Statistical Analysis}

In unmatched analyses, descriptive statistics comparing cases and controls were calculated for demographic information, including age, race, marital status, education, employment, income, and center of CrMS, along with medication use and menstrual cycle function. For cases, length taking OCs and time since discontinuation of OCs were also calculated. Exact chi-square and the Student's $t$ test were used to calculate differences between recent $\mathrm{OC}$ users and controls on demographic and clinical characteristics and, for the first cycle, of biomarkers of menstrual cycle function.

In matched analyses, linear mixed models using robust standard errors (SE) were used to evaluate differences in menstrual cycle function (described in detail below). These randomintercept models were chosen to account for the correlation between cycles of the matched pairs. $P$ values were 2-sided with significance set at 0.05 .

The choice of covariates to adjust for in the mixed models was determined by both clinical knowledge and bivariate statistical testing. Medications were initially assessed as possible confounders of mucus score, as use could be related to the exposure (recent OC users 
vs. controls) and independently related to the outcome, and center of CrMS instruction was assessed for potential confounding of all outcomes. Variables that were significant at the $p<$ 0.10 level in bivariate analyses (e.g., difference in luteal phase length $=$ intercept + potential confounder) were included in the adjusted models. Additionally, any medications that were significant at the $p<0.10$ level for mucus score were assessed for potential confounding with the other outcomes. Statistical tests revealed that only vitamin use among controls was a potential confounder for mucus score, and center of CrMS instruction was a potential confounder for menstrual flow score.

Because neither exposure nor covariates were time varying, nonweighted models were deemed appropriate. Due to the limited number of independent variables (none of which were continuous), checking for linearity, interactions, and multicollinearity was not necessary. Models for each of the six outcomes were assessed via residual plots, histograms of the residuals to check for normality and examination of potential outliers. SAS (version 9.2, SAS Institute Inc, Cary, NC) was used for all data analyses.

\section{Definitions}

\section{Cycle Length}

The number of days from the first day of menses (as recorded on the CrMS chart by a woman) to the last day of the cycle before the beginning of the next menses.

\section{Estimated Day of Ovulation (EDO)}

For this study, the number of days counted from the first day of menstrual flow through the peak day of cervical mucus, the latter of which was validated retrospectively by professional CrMS teachers.

\section{Length of Luteal Phase}

The number of days counted from the first day after the EDO through the last day of the menstrual cycle.

\section{Length of Menses}

The number of days of continuous usual bleeding at the beginning of a menstrual cycle as charted by the participant. 


\section{Mucus Score}

This is an index developed by Hilgers ${ }^{19}$ to estimate the quality of the cervical mucus of each day within the fertile window (i.e., EDO and the 5 days before it). The system takes into consideration the color, consistency, sensation, and change of the cervical mucus, giving a numerical rate of $0-4$ for each of the parameters on each day. The score on each day ranges from 0 to 16. The scores for the 6 days of the fertile window are averaged. A regular cervical mucus score ranges from 9.1 to 16 , with the maximum score being 16 .

\section{Menstrual Flow Score}

This is an index developed by Hilgers ${ }^{18}$ as a qualitative estimate of the intensity of menstrual flow. The index is based on points assigned for a woman recording each day of heavy bleeding (12 points), moderate bleeding (8 points), light bleeding (4 points), very light bleeding ( 2 points), brown bleeding ( 1 point) and takes the mean of the points assigned each day for the first 6 days of the menstrual cycle.

\section{Results}

\section{Demographics}

There were no statistically significant differences in the mean age, race, education, employment status, income, or center of CrMS study between the recent OC users and controls (Table 2). Recent OC users were more likely to be married than controls $(p<0.0001)$ and have incomes $>\$ 40,000(p=0.01)$.

\section{Satisfaction and Confidence in Using the CrMS}

There were no significant differences in either satisfaction with or confidence in using the CrMS between cases and controls (both for the women and their partners) after both the first and sixth follow-up visits (Table 3 ).

\section{Menstrual Cycle Characteristics}

Descriptives of menstrual cycle characteristics in unmatched, unadjusted analyses for the first cycle after beginning CrMS instruction are shown in Table 2. Controls had longer duration of menses (6.2 vs. 5.5 days, $p=0.004$ ) along with higher scores for intensity of menstrual flow (6.2 vs. $4.9, p=0.0001)$ and mucus quality (9.0 vs. $7.3, p=0.01$ ).

As shown in Table 4, matched pair analyses assessed the difference in menstrual cycle biomarkers for the first two cycles combined and the first six cycles combined after beginning 
CrMS instruction. For the first two cycles, significant differences were found for recent OC users for menses duration (shorter) and estimated day of ovulation (later). Similar trends were seen when looking at the six combined cycles after beginning CrMS, with significant differences found for case length of the cycle (longer) and EDO (later). Both mucus and menses cycle score differences were significantly lower for recent OC users compared to controls for the first two and six consecutive cycles $(p<0.005)$. In cycle-specific analyses, the difference for the mucus score persisted but was not statistically significant for individual menstrual cycles after the second cycle (Fig. 2). The difference for the menses score was attenuated and no longer statistically significant after the fourth cycle (Fig. 3). The difference in the estimated day of ovulation seemed to persist but was significant only for the individual second cycle (Fig. 4). Neither restricting all six outcome analyses to women who had cycle lengths $<60$ days nor deleting observations with residuals in the first or last percentiles significantly altered the results.

For the recent OC users, on average, there was a 23.9 day gap (range 0-77 days) between discontinuing OCs and the beginning of CrMS charting. To investigate whether women who had a longer gap influenced the results, we repeated the analyses excluding all matched pairs with women who had stopped OCs for $\geq 30$ days before starting to chart with the CrMS ( $n=$ 156). The results were all substantively similar to those we have reported, except that confidence intervals (Cls) were wider because of the smaller sample size (data not shown).

\section{Discussion}

After discontinuation of OCs, women on average will experience longer cycles as well as more variability in cycle length and EDOthanwomen who have not recently used OCs. A similar finding was observed by Rice-Wray et al. ${ }^{3}$ and by Larsson-Cohn ${ }^{4}$ in their studies of menstrual cycle after discontinuation of OCs. The average menstrual cycle length for non-OC users has been reported to be $28.9 \pm 3.4$ days. This value is in agreement with the average menstrual cycle length for our control group $29.8 \pm 6.9$ days; however, the recent users of OCs had an average menstrual cycle length of $31.5 \pm 11.1$ days (our study) and 36 days in other studies, which are well above the value reported for non-OC users. ${ }^{3,4}$ Gnoth et al. ${ }^{5}$ also reported an increase in the average menstrual cycle length. The average menstrual cycle lengths in the first three cycles of their study were 33.3 days after OC discontinuation compared with 29.6 days for non-OC users, which is in agreement with our study. This prolongation of the menstrual cycle post-OCs is thought to be due to the time needed for the hypothalamuspituitary- ovarian axis to normalize after its suppression secondary to the intake of OCs and is reflected in longer follicular phases. On the other hand, Duijkers et al. ${ }^{20}$ have reported a normal duration of the first 
cycle after discontinuation of OCs as compared with womenwho were not taking OCs, that is, 30 days and 29 days, respectively.

In our study, the number of days to EDO (follicular phase) for the first cycle post-OC discontinuation was $20.4 \pm 11.4$ and $18.5 \pm 6.7$ days for the case and control groups, respectively. Birtch et al. ${ }^{21}$ reported the length of the preovulatory phase of the first menstrual cycle after OC discontinuation as being $20.5 \pm 1.0$ days, which is in good agreement with our study group. In addition, a study from Jukic et al. ${ }^{22}$ showed that women with recent use of OCs had a longer follicular phase by a mean of 2.3 days compared to those women who did not use OCs. This finding is in close agreement with our study, where an increase of approximately 2 days was observed among the post-OC group compared to the control group. The duration of the follicular and luteal phases for the control group in cycle 1 (18.5 and 11.5 days, respectively) is slightly longer and shorter, respectively, than the durations reported in other recent studies with roughly similar age distributions of women. A European study of 782 women and 6724 cycles reported a mean follicular phase length of 16.3 days and a mean luteal phase length of 12.7 days, ${ }^{23}$ whereas in the study by Gnoth et al. ${ }^{5}$ the first cycle in the control group $(n=254)$ had a mean follicular phase length of 17.0 days and a mean luteal phase length of 12.1 days.

The presence of high-quality cervical mucus during a woman's menstrual cycle is a very important factor for a couple attempting to achieve pregnancy because cervical mucus enhances sperm survival and transport. ${ }^{24}$ It is of interest that the mean cervical mucus scores for both the recent OC users (9.0) and control women (7.3) fell below the range of a regular mucus cycle score (9.1-16). ${ }^{19}$ The normal variability of the cervical mucus score with other factors, such as age and parity, has not been systematically assessed. The probability of pregnancy with intercourse on days with no cervical secretion is near zero. ${ }^{25}$ It has also been shown that a lower quality of mucus discharge as assessed with different measures, including the CrMS mucus score, correlates with a lower probability of conception. ${ }^{14,24,26}$ Fehring $^{16}$ showed that $97.8 \%$ of the peak day mucus fell within \pm 4 days of the LH surge within a menstrual cycle. In addition, Stanford et al. ${ }^{13}$ provided evidence that the maximum probability of conception differs significantly by mucus quality, as assessed by the CrMS mucus score; a mucus score change of 2 corresponds to an absolute change in the per cycle probability of conception of about $5 \%$. The presence of peak day mucus correlates well with the rising of estrogen levels in the blood. ${ }^{18}$ Our study showed that not only for the first but also for the combined first six menstrual cycles after discontinuation of OCs the quality of the cervical mucus was diminished compared with that of the control group. We know of no other study that has reported on the cervical mucus quality after $\mathrm{OC}$ discontinuation. 
We also found that recent $\mathrm{OC}$ users had decreased menstrual flow, as assessed by duration of menses and the menstrual flow score, which measures menstrual intensity based on the woman's subjective assessment. It is well known that OCs decrease the length and flow of menses (OC withdrawal bleeding) by $\geq 60 \%$ during their use. ${ }^{27}$ This decrease in menses flow and length is most likely caused by a substantial thinning of the endometrium thickness in women taking OCs. ${ }^{28}$ Therefore, the initial decrease in the length of the menses and menstrual flow score in women discontinuing OCs is not surprising. Our results suggest that it takes the endometrium at least six cycles after OC use to return to a normal intensity of flow. A thinner endometrium has been shown to be correlated with a lower likelihood of implantation in the setting of assisted reproduction. ${ }^{29}$

\section{Limitations}

The OC effects on menstrual cycle biomarkers might endure for up to 9 months, as reported by Gnoth et al. ${ }^{5}$ but by design, our study assessed only the first six cycles and had limited power because of the decreasing number of contributing cycles at each cycle number. The lack of statistical power for individual cycles limits our ability to define the exact duration of the continued effects of prior OC use. Whereas some parameters clearly attenuated with time (i.e., the menses score) (Fig. 3), others seemed to continue to have differences of similar magnitude across cycles (i.e., the mucus score and the EDO) (Figs. 2 and 4). In these latter cases, the combined analysis of cycles 1-6 is appropriate to increase the power of the analysis (Table 4 ) by combining multiple cycles.

More precise measures of when menstrual cycle function returns to normal are necessary, but our results corroborate other studies that show a delay of return to full fecundity. This initial reduction in fecundity could be a result of mistimed intercourse because of delayed fertile phases, decreased quality of cervical mucus adversely impacting sperm survival, decreased thickness of the endometrium lowering the probability of implantation, or a combination of these factors. Our study was a retrospective study but was based on clinical records that were completed prospectively at the time the women were beginning use of the CrMS. Our careful matching process seems to have compensated for possible confounding variables of age and parity. A prospectively conducted study would likely have a very similar design but could include additional helpful biomarkers, such as hormonal measures. Conducting a prospective randomized clinical trial for this question would be ethically problematic because it would require randomizing women to take OCs and then stop taking them. 
We cannot exclude the possibility that some of the women who are recent OC users were initially placed in the $\mathrm{OC}$ to regulate their otherwise irregular menstrual cycles. After the discontinuation of OCs, such women may return to a pattern of irregular menstrual cycles, which could have contributed to the large variability in the standard deviation in the menstrual cycle length. However, there was no exclusion by cycle length in our study, such that women with longer and irregular menstrual cycles were included in the control group. Limiting the analysis to women who had cycles $<60$ days (in both the OC and control groups) did not substantially alter our results. Another limitation is that cervical mucus is an imperfect means of identifying the exact day of ovulation. However, any variability introduced by this indicator for ovulation would not be expected to differ systematically between women who are recent OC users and women who are not. In our sample, recent OC users were more likely to be married and have higher income. It is possible that they would, therefore, be more likely interested in achieving pregnancy, but it is difficult to predict what relationship this would have with cycle parameters. Marriage might be associated with more or less stress, and higher income might be associated with less overweight (more regular cycles) or more consistent vigorous exercise (possibly more irregular cycles). Although race and ethnicity are not thought to directly affect menstrual cycle function, future studies involving women from various racial=ethnic backgrounds are recommended. Some of the women in the control group may have used OCs $>1$ year before the study. Although the effects of past use of OCs are not thought to last for more than 12 months, future studies should clarify and control for a woman's complete history of OC use. Finally, the types of OCs in use during the time of this study were generally of somewhat higher dose than the types of OCs in use today. It will be important to replicate this study with current formulations of OCs.

In spite of these limitations, our results offer potential explanatory mechanisms for the Wiegratz et al. study, ${ }^{30}$ which showed a $15 \%$ decrease in conception rate in the first 3 months of the discontinuation of OCs. In addition, Birtch et al. ${ }^{31}$ found clinically low levels of progesterone in $40 \%$ of the cycles after discontinuation of OCs. They hypothesize that low levels of progesterone could result from luteal phase dysfunction, which would further explain the delay in fertility on post-OC women.

For those couples who wish to avoid pregnancy with the CrMS after discontinuing OCs, the similar confidence and satisfaction scores found in this study are reassuring. Previous work has demonstrated that the effectiveness of the CrMS to avoid pregnancy is equally good for those discontinuing OCs as for those with no recent use of hormonal contraception. ${ }^{25}$ For those couples who wish to conceive after discontinuing OCs, practitioners can use this information to 
ease couples' anxiety about delays in achieving pregnancy after discontinuation of OCs. In addition, they can provide couples with useful information on how to increase their chances to achieve pregnancy, such as learning how to determine their fertile window through the use of natural biomarkers (especially biomarkers that prospectively identify the fertile window: cervical mucus or levels of urinary estrogen) and to focus intercourse during the estimated fertile window. ${ }^{7,14}$ Gnoth et al. ${ }^{32}$ found that by using timed intercourse during the estimated fertile phase, $88 \%$ of fertile couples (including couples discontinuing OCs) can achieve pregnancy within 6 months.

\section{Conclusions}

Statistically significant differences in several biomarkers of the women's menstrual cycle were observed after discontinuation of OCs. These changes may help explain why there is a decrease in the chances for a couple to conceive within the first few months after discontinuation of OCs. There is a trend toward normalization of some of these parameters over the first six menstrual cycles of discontinuation of OCs. Confidence and satisfaction in use of the CrMS did not differ between women discontinuing OCs and those not using OCs.

\section{Acknowledgments}

We acknowledge Xiaoming Sheng, Ph.D., for statistical consultation; Kathleen Raviele, M.D., for contributing CrMS charts; and Maria Wellenstein, M.S.N., who was instrumental in collecting charts and developing the dataset. C.L.N. would like to thank Mayo Clinic Internal Medicine Residency Program for approving the proposal and granting the opportunity for this research experience. We thank the CrMS-trained professionals who helped with data abstracting and checking, including Ann M. Prebil, R.N., B.S.N.; Jeanne F. Stoll, R.N.; Lucy M. Harris, R.N., B.S.N.; Marilyn Clayton, R.N., and Patricia A. Rapplean, R.N., B.S.N. We thank Xiangyang Ye, Senior Research Analyst at the University of Utah Pharmacotherapy Outcomes Research Center, who provided the SAS macro for random matching of the cases and controls. Finally, this study was partially funded by the University of Utah, Department of Family and Preventive Medicine, Health Studies Fund, and the University of Missouri-Columbia, Department of Family and Community Medicine.

\section{Disclosure Statement}

No competing financial interests exist. 


\section{References}

1. Mosher WD, Martinez GM, Chandra A, Abma JC, Willson SJ. Use of contraception and use of family planning services in the United States: 1982-2002. Adv Data 2004(350):136.

2. Stanford JB, Lemaire JC, Thurman PB. Women's interest in natural family planning. J Fam Pract 1998;46:65-71.

3. Rice-Wray E, Correu S, Gorodovsky J, Esquivel J, Goldzieher JW. Return of ovulation after discontinuance of oral contraceptives. Fertil Steril 1967;18:212-218.

4. Larsson-Cohn $U$. The length of the first three menstrual cycles after combined oral contraceptive treatment. Acta Obstet Gynecol Scand 1969;48:416-422.

5. Gnoth C, Frank-Herrmann P, Schmoll A, Godehardt E, Freundl G. Cycle characteristics after discontinuation of oral contraceptives. Gynecol Endocrinol 2002;16:307-317.

6. Robinson JE, Wakelin M, Ellis JE. Increased pregnancy rate with use of the Clearblue Easy Fertility Monitor. Fertil Steril 2007;87:329-334.

7. Scarpa B, Dunson DB, Giacchi E. Bayesian selection of optimal rules for timing intercourse to conceive by using calendar and mucus. Fertil Steril 2007;88:915-924.

8. Wilcox AJ, Weinberg CR, Baird DD. Timing of sexual intercourse in relation to ovulation. Effects on probability of conception, survival of the pregnancy, and sex of baby. $\mathrm{N}$ Engl $\mathrm{J}$ Med 1995;333:1517-1521.

9. Eriksen GV, Carlstedt I, Uldbjerg N, Ernst E. Cervical mucins affect the motility of human spermatozoa in vitro. Fertil Steril 1998;70:350-354.

10. Odeblad E. The functional structure of human cervical mucus. Acta Obstet Gynecol Scand 1968:47(Suppl 1):57-79.

11. Odeblad E. Cervical mucus and their functions. J Ir Coll Phys Surg 1997;26:27-32.

12. Menarguez M, Pastor LM, Odeblad E. Morphological characterization of different human cervical mucus types using light and scanning electron microscopy. Hum Reprod 2003;18:1782-1789.

13. Stanford JB, Smith KR, Dunson DB. Vulvar mucus observations and the probability of pregnancy. Obstet Gynecol. 2003;101:1285-1293.

14. Stanford JB, White GL, Hatasaka H. Timing intercourse to achieve pregnancy: Current evidence. Obstet Gynecol 2002; 100:1333-1341.

15. Hilgers TW. Introduction to the Creighton Model System. In: Hilgers TW, ed. The medical and surgical practice of Na- ProTechnology. Omaha, NE: Pope Paul VI Institute Press; 2004:43-56. 
16. Fehring RJ. Accuracy of the peak day of cervical mucus as a biological marker of fertility. Contraception 2002;66: 231-235.

17. Ecochard R, Boehringer $\mathrm{H}$, Rabilloud M, Marret H. Chronological aspects of ultrasonic, hormonal, and other indirect indices of ovulation. Br J Obstet Gynaecol 2001;108:822829.

18. Hilgers TW. Scientific foundations of the CrMS. In: Hilgers TW, ed. The medical and surgical practice of NaPro-Technology. Omaha, NE: Pope Paul VI Institute Press; 2004:183-214.

19. Hilgers TW. Objective classification of the mucus cycle. In: Hilgers TW, ed. The medical and surgical practice of NaProTechnology. Omaha, NE: Pope Paul VI Institute Press; 2004:177-182.

20. Duijkers I, Engels L, Klipping C. Length of the menstrual cycle after discontinuation of oral contraceptives. Gynecol Endocrinol 2005;20:74-79.

21. Birtch RL, Baerwald AR, Olatunbosun OA, Pierson RA. Ultrasound image attributes of human ovarian dominant follicles during natural and oral contraceptive cycles. Reprod Biol Endocrinol 2005;3:12.

22. Jukic AM, Weinberg CR, Baird DD, Wilcox AJ. Lifestyle and reproductive factors associated with follicular phase length. J Womens Health 2007;16:1340-1347.

23. Colombo B, Masarotto G. Daily fecundability: First results from a new data base. Demogr Res 2000;3:5.

24. Bigelow JL, Dunson DB, Stanford JB, Ecochard R, Gnoth C, Colombo B. Mucus observations in the fertile window: A better predictor of conception than timing of intercourse. Hum Reprod 2004;19:889-892.

25. Howard MP, Stanford JB. Pregnancy probabilities during use of the Creighton Model FertilityCare System. Arch Fam Med 1999;8:391-402.

26. Scarpa B, Dunson DB, Colombo B. Cervical mucus secretions on the day of intercourse: An accurate marker of highly fertile days. Eur J Obstet Gynecol Reprod Biol 2006;125:72-78.

27. Hatcher RA, Trussell J, Stewart F, et al. Contraceptive technology, 18th ed. New York: Ardent Media, Inc., 2004.

28. Grow DR, Iromloo K. Oral contraceptives maintain a very thin endometrium before operative hysteroscopy. Fertil Steril 2006;85:204-207.

29. Oliveira JB, Baruffi RL, Mauri AL, Petersen CG, Borges MC, Franco JG Jr. Endometrial ultrasonography as a predictor of pregnancy in an in-vitro fertilization programme after 
ovarian stimulation and gonadotrophin-releasing hormone and gonadotrophins. Hum Reprod 1997;12:2515-2518.

30. Wiegratz I, Mittmann K, Dietrich H, Zimmermann T, Kuhl H. Fertility after discontinuation of treatment with an oral contraceptive containing 30 microg of ethinyl estradiol and $2 \mathrm{mg}$ of dienogest. Fertil Steril 2006;85:1812-1819.

31. Birtch RL, Olatunbosun OA, Pierson RA. Ovarian follicular dynamics during conventional vs. continuous oral contraceptive use. Contraception 2006;73:235-243.

32. Gnoth C, Godehardt D, Godehardt E, Frank-Herrmann P, Freundl G. Time to pregnancy: Results of the German prospective study and impact on the management of infertility. Hum Reprod 2003;18:1959-1966. 


\section{Appendix}

Fig. 1: Creighton Model FertilityCare Sysetm (CrMS) Example Chart (Courtesy of T. W. Hilgers, Pope Paul VI Institute, Omaha, NE.)

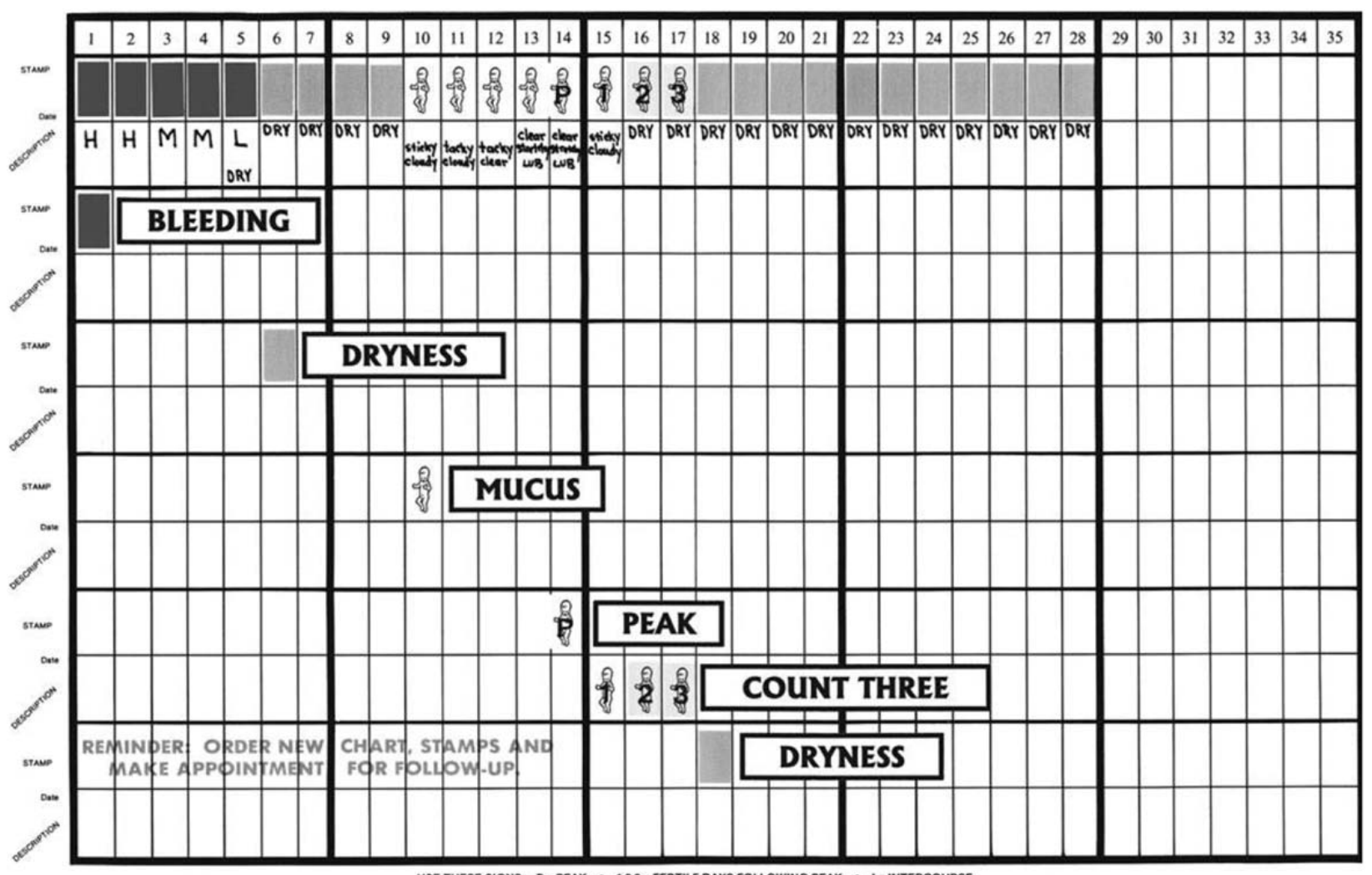


Fig. 2

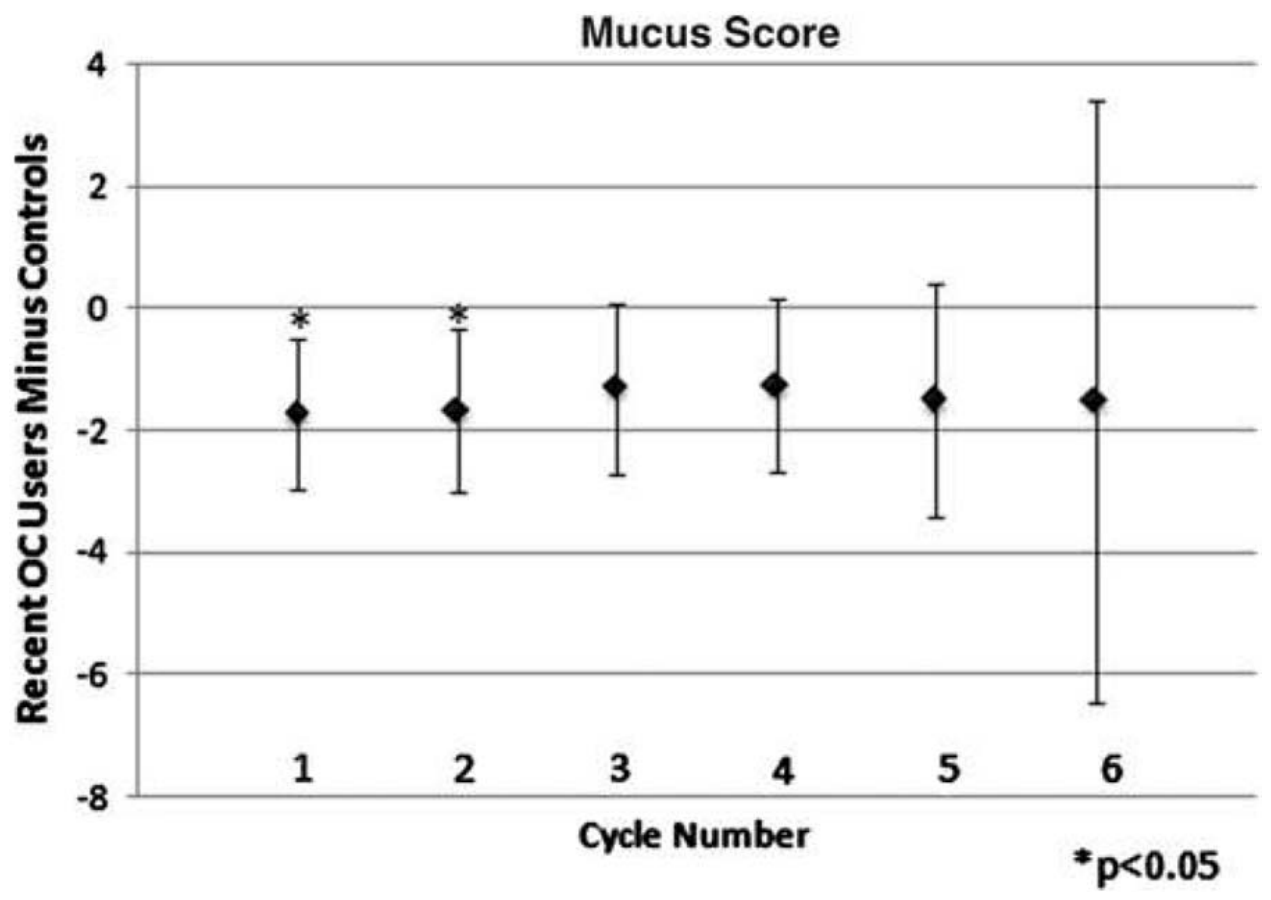

Mean difference in mucus score between recent oral contraceptive $(\mathrm{OC})$ users and controls, adjusted forv itamin use by controls (positive indicates that recent $\mathrm{OC}$ users have the larger value). Number of matched pairs in each cycle can be found in Table 1. 
Fig. 3

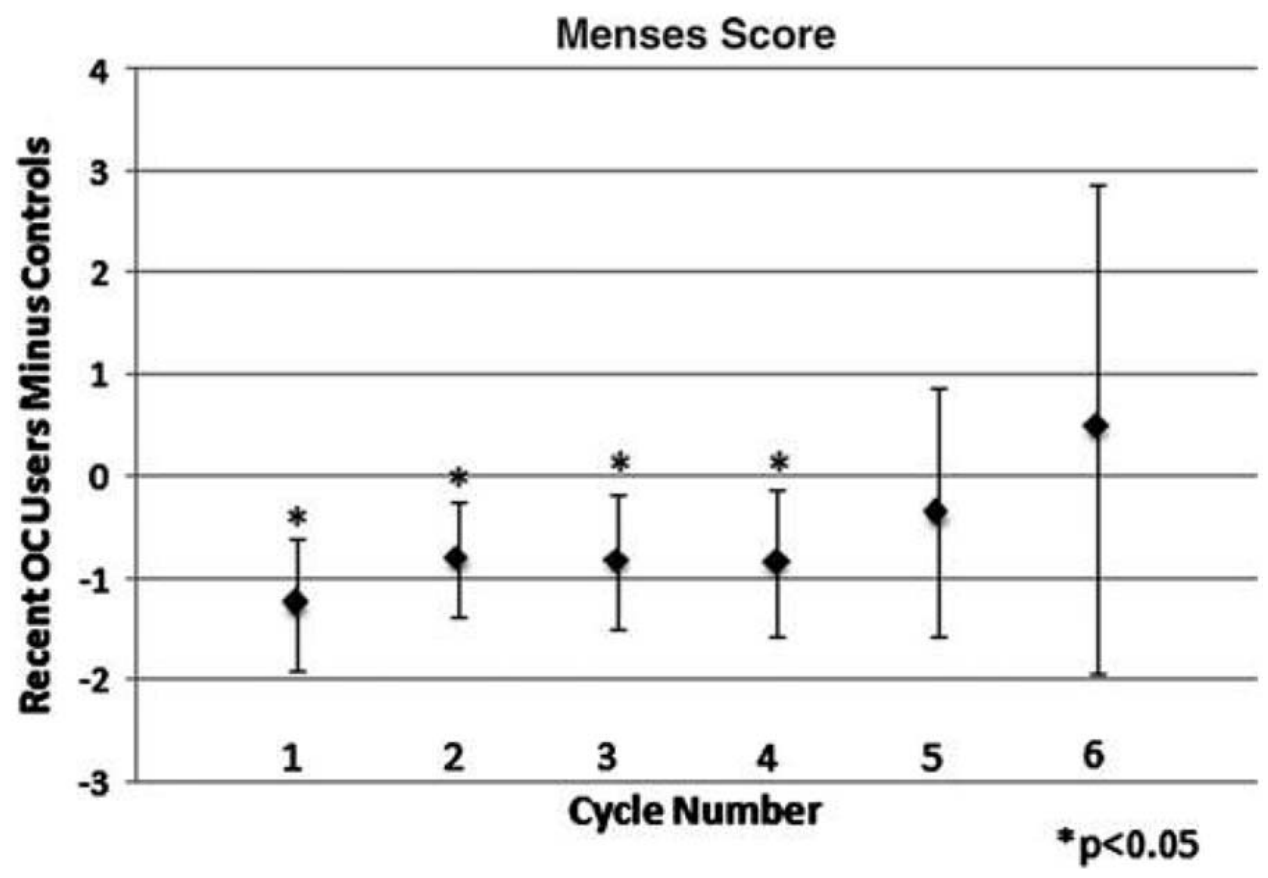

Mean difference in menses score between recent OC users and controls, adjusted for CrMS center of instruction (positive indicates that recent OC users have the larger value). Number of matched pairs in each cycle can be found in Table 1. 
Fig. 4

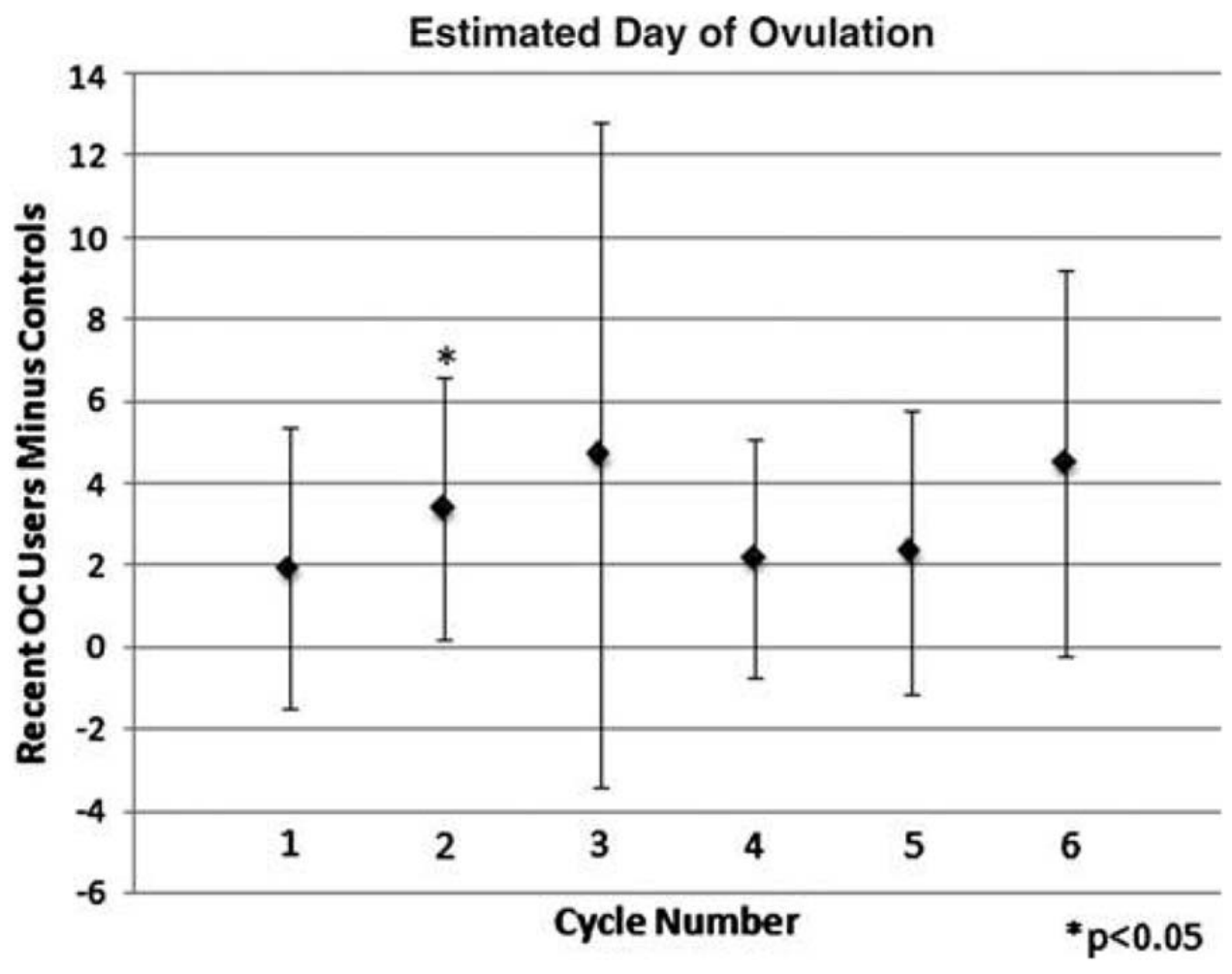

Mean difference in estimated day of ovulation between recent $\mathrm{OC}$ users and controls (positive indicates that recent $\mathrm{OC}$ users have the latter day). Number of matched pairs in each cycle can be found in Table 1. 
Table 1: Matched Pairs of Cycle by Cycle Number: Recent Oral Contraceptive Users Matched with Controls

\begin{tabular}{lc}
\hline Cycle number & Total pairs $(\mathrm{n}=230)$ \\
\hline 1 & 66 \\
2 & 55 \\
3 & 44 \\
4 & 36 \\
5 & 22 \\
6 & 7 \\
\hline
\end{tabular}




\section{Table 2: Characteristics of Study Population According to Group}

\begin{tabular}{|c|c|c|c|c|}
\hline Characteristic & Recent OC users $(\mathrm{n}=70)$ & Controls $(\mathrm{n}=70)$ & Total $(\mathrm{n}=140)$ & $\mathrm{p}$ value \\
\hline \multicolumn{5}{|l|}{ Demographics } \\
\hline Age, mean $\pm S D$ & $\begin{array}{l}26.2 \pm 3.2 \\
\mathrm{n}(\%)\end{array}$ & $\begin{array}{c}26.6 \pm 3.3 \\
\mathrm{n}(\%)\end{array}$ & $\begin{array}{l}26.4 \pm 3.2 \\
\mathrm{n}(\%)\end{array}$ & 0.48 \\
\hline Previously had a live birth & $7(10.0)$ & $7(10.0)$ & $14(10.0)$ & 1.00 \\
\hline \multicolumn{5}{|l|}{ Race } \\
\hline White & $68(97.1)$ & $67(95.7)$ & $135(96.4)$ & 1.00 \\
\hline Other & $2(2.9)$ & $3(4.3)$ & $5(3.6)$ & \\
\hline \multicolumn{5}{|l|}{ Marital status } \\
\hline Single/divorced & $4(5.7)$ & $13(18.6)$ & $17(12.1)$ & $<0.0001$ \\
\hline Engaged & $10(14.3)$ & $35(50.0)$ & $45(32.1)$ & \\
\hline Married & $56(80.0)$ & $22(31.4)$ & $78(55.7)$ & \\
\hline \multicolumn{5}{|l|}{ Education } \\
\hline High school/vocational & $7(10.0)$ & $13(18.6)$ & $20(14.3)$ & 0.14 \\
\hline Some college & $16(22.9)$ & $7(10.0)$ & $23(16.4)$ & \\
\hline Completed college & $36(51.4)$ & $39(55.7)$ & $75(53.6)$ & \\
\hline Graduate school & $11(15.7)$ & $11(15.7)$ & $22(15.7)$ & \\
\hline Employed & $62(88.6)$ & $59(84.3)$ & $121(86.4)$ & 0.46 \\
\hline \multicolumn{5}{|l|}{ Income } \\
\hline$<40,000$ & $25(35.7)$ & $40(57.1)$ & $65(46.4)$ & 0.01 \\
\hline$\geq 40,000$ & $45(64.3)$ & $30(42.9)$ & $75(53.6)$ & \\
\hline \multicolumn{5}{|l|}{ Center } \\
\hline Georgia & $13(18.6)$ & $10(14.3)$ & $23(16.4)$ & 0.79 \\
\hline Missouri & $41(58.6)$ & $43(61.4)$ & $84(60.0)$ & \\
\hline Wisconsin & $16(22.9)$ & $17(24.3)$ & $33(23.6)$ & \\
\hline \multicolumn{5}{|l|}{ Medications } \\
\hline Antibiotics & $3(4.3)$ & $13(18.6)$ & $16(11.4)$ & 0.01 \\
\hline Antihistamines & $15(21.4)$ & $11(15.7)$ & $27(19.3)$ & 0.38 \\
\hline Vitamins & $25(35.7)$ & $20(28.6)$ & $45(32.1)$ & 0.37 \\
\hline Expectorants & $2(2.9)$ & $2(2.9)$ & $4(2.9)$ & 1.00 \\
\hline Hormones & $1(1.4)$ & $4(5.7)$ & $5(3.6)$ & 0.37 \\
\hline $\begin{array}{l}\text { Menstrual function for first } \\
\text { cycle after starting CrMS }\end{array}$ & Mean $\pm S D$ & Mean \pm SD & Mean \pm SD & \\
\hline Length of luteal phase (days) & $11.1(3.0)$ & $11.5(2.6)$ & $11.3(2.8)$ & 0.39 \\
\hline Length of menstrual flow (days) & $5.5(1.5)$ & $6.2(1.2)$ & $5.9(1.4)$ & 0.004 \\
\hline Length of cycle (days) & $31.5(11.1)$ & $29.8(6.9)$ & $30.6(9.2)$ & 0.32 \\
\hline Estimated day of ovulation & $20.4(11.4)$ & $18.5(6.7)$ & $19.4(9.4)$ & 0.25 \\
\hline Menstrual flow score & $4.9(2.1)$ & $6.2(1.4)$ & $5.59(1.9)$ & 0.0001 \\
\hline Cervical mucus score & $7.3(4.0)$ & $9.0(3.5)$ & $8.12(3.8)$ & 0.01 \\
\hline
\end{tabular}

${ }^{a}$ Due to four matched pairs with a cycle 1, total number of women analyzed was 132.

CrMS, Creighton Model Fertility Care System; OC, oral contraceptives; SD, standard deviation. 


\section{Table 3: Satisfaction and Confidence with CrMS According to Group (Scale}

\section{of 1-5)}

\begin{tabular}{|c|c|c|c|c|c|}
\hline & $\mathrm{n}$ & Recent OC users & Control & Total & $\mathrm{p}$ value \\
\hline Satisfaction & & $($ mean $\pm S D)$ & $($ mean $\pm S D)$ & $($ mean $\pm S D)$ & \\
\hline Woman's satisfaction after 1st follow-up & 134 & $3.9(0.91)$ & $4.0(0.66)$ & $3.9(0.78)$ & 0.58 \\
\hline Woman's satisfaction after 6th follow-up & 112 & $4.4(0.71)$ & $4.4(0.56)$ & $4.4(0.63)$ & 0.83 \\
\hline Man's satisfaction after 1st follow-up & 107 & $3.8(0.74)$ & $3.8(0.84)$ & $3.8(0.79)$ & 0.73 \\
\hline Man's satisfaction after 6th follow-up & 69 & $4.4(0.61)$ & $4.2(0.63)$ & $4.3(0.62)$ & 0.27 \\
\hline \multicolumn{6}{|l|}{ Confidence } \\
\hline Woman's confidence after 1st follow-up & 134 & $3.3(0.86)$ & $3.4(0.91)$ & $3.4(0.89)$ & 0.86 \\
\hline Woman's confidence after 6th follow-up & 111 & $4.3(0.72)$ & $4.3(0.59)$ & $4.3(0.66)$ & 0.77 \\
\hline Man's confidence after 1st follow-up & 106 & $3.3(0.82)$ & $3.6(0.77)$ & $3.4(0.81)$ & 0.08 \\
\hline Man's confidence after 6 th follow-up & 72 & $4.3(0.72)$ & $4.3(0.58)$ & $4.3(0.64)$ & 0.76 \\
\hline
\end{tabular}


Table 4: Adjusted Mean Difference in Cycle Parameters between Recent Oral Contraceptive Users and Controls

\begin{tabular}{lccc}
\hline Outcome & Cycles & $\mathrm{n}$ & Mean difference $(C I)$ \\
\hline $\begin{array}{c}\text { Length of luteal } \\
\text { phase (days) }\end{array}$ & $1-2$ & 121 & $-0.55(-1.44-0.34)$ \\
$\quad 1-6$ & 230 & $-0.13(-1.03-0.78)$ \\
$\begin{array}{c}\text { Length of menses } \\
\text { (days) }\end{array}$ & $1-2$ & 120 & $-0.48(-0.86--0.11)^{*}$ \\
$\quad 1-6$ & 229 & $-0.30(-0.67-0.07)$ \\
$\begin{array}{l}\text { Length of cycle } \\
\quad \text { (days) }\end{array}$ & $1-2$ & 121 & $2.51(-0.36-5.39)$ \\
$\begin{array}{c}\text { Estimated day } \\
\text { of ovulation }\end{array}$ & $1-6$ & 230 & $3.50(0.14-6.87)^{*}$ \\
$\quad 1-2$ & 121 & $3.00(0.02-5.97)^{*}$ \\
$\begin{array}{c}\text { Menstrual flow } \\
\text { score }\end{array}$ & $1-6$ & 226 & $3.78(0.27-7.30)^{*}$ \\
Cervical mucus $_{\text {score }}^{\mathrm{b}}$ & $1-6$ & 228 & $-1.15(-1.66--0.63)^{* *}$ \\
& $1-2$ & 120 & $-2.36(-3.64--1.09)^{* *}$ \\
\hline
\end{tabular}

Positive means recent $\mathrm{OC}$ users have the larger value or later day. ${ }^{*} p<0.05 ;{ }^{* *} p<0.005$.

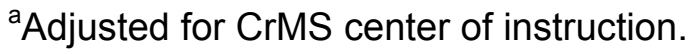

${ }^{\mathrm{b}}$ Adjusted for vitamin use by controls.

$\mathrm{Cl}$, confidence interval. 